\title{
Data-Weighting Periodic RLS Based Adaptive Control Design and Analysis without Linear Growth Condition
}

\author{
Ronghu Chi, ${ }^{1}$ Zhongsheng Hou, ${ }^{2}$ and Shangtai Jin ${ }^{2}$ \\ ${ }^{1}$ School of Automation \& Electronic Engineering, Qingdao University of Science \& Technology, Qingdao 266042, China \\ ${ }^{2}$ Advanced Control Systems Lab, School of Electronics \& Information Engineering, Beijing Jiaotong University, Beijing 100044, China \\ Correspondence should be addressed to Ronghu Chi; ronghu_chi@hotmail.com
}

Received 5 March 2014; Accepted 27 June 2014; Published 10 July 2014

Academic Editor: Nazim I. Mahmudov

Copyright (c) 2014 Ronghu Chi et al. This is an open access article distributed under the Creative Commons Attribution License, which permits unrestricted use, distribution, and reproduction in any medium, provided the original work is properly cited.

\begin{abstract}
A new periodic recursive least-squares (PRLS) estimator is developed with data-weighting factors for a class of linear time-varying parametric systems where the uncertain parameters are periodic with a known periodicity. The periodical time-varying parameter can be regarded as a constant in the time interval of a periodicity. Then the proposed PRLS estimates the unknown time-varying parameter from period to period in batches. By using equivalent feedback principle, the feedback control law is constructed for the adaptive control. Another distinct feature of the proposed PRLS-based adaptive control is that the controller design and analysis are done via Lyapunov technology without any linear growth conditions imposed on the nonlinearities of the control plant. Simulation results further confirm the effectiveness of the presented approach.
\end{abstract}

\section{Introduction}

Repetitive control (RC), introduced by Inoue et al. [1, 2] originally, is an effective control scheme for tracking periodic reference and rejecting periodic disturbance signals. It is regarded as a simple learning controller and the control input is calculated using the information of the error signal in the preceding periods. The basic theory and convergence analysis were shown in the pioneering works [3-5]. The necessary and sufficient conditions for asymptotic stability of the continuous-time repetitive controller were restrictively formulated in $[6]$. In $[7,8]$, the stability of repetitive controllers is analyzed in the discrete-time domain. To enhance the robustness of these repetitive control schemes, researchers in $[4,7]$ modified the repetitive update rule to include the socalled Q-filter.

However, the analysis and design of repetitive control are mainly performed in the frequency domain [1-8], which makes the nonlinear study more difficult. Most of the stability results in repetitive control require that the dynamic system be assumed linear or it could be as least partially linearized with feedback control. As stated in [9], learning controllers could be synthesized and analyzed using a similar approach to the adaptive control. Adaptive control [10-15] of nonlinear systems has been an area of increasing research activity and global regulation and tracking results have been obtained for several classes of nonlinear systems. However, as indicated in [15], no adaptive control algorithms developed hitherto can solve unknown parameters with arbitrarily fast and nonvanishing variations.

It is worth pointing out that periodic variations are encountered in many real systems. These variations can exist in the system parameters [16], or as a disturbance to the system [17]. When the periodicity of system parameters is known a priori, some new adaptive controllers with periodic updating have been constructed by means of a pointwise integral mechanism $[16,18-20]$. However, only a few results of discrete-time periodic adaptive control [21-23] were proposed and they have to impose linear growth conditions on the nonlinearities to provide global stability.

Note that repetitive control is closely related to iterative learning control [24], which is developed for control tasks that repeat in a finite duration with perfect tracking requirement. For example, a no-reset ILC approach was proposed in [25] because the system never actually starts, stops, resets, and then repeats, and a new nonstandard ILC algorithm was 
used in [26] for tracking periodic signals by defining a "trial" in terms of completion of a single "period" of the output trajectory. More recently, Chi et al. [27] proposed a discretetime adaptive ILC (AILC), where the parameters are updated in the iteration domain. Further a nonlinear data-weighted iterative recursive least-squares algorithm [28] was developed to extend the discrete-time AILC to linear parametric systems without linear growth condition.

Motivated by the above discussion, this paper uses the formalism of discrete-time AILC $[27,28]$ to solve the repetitive control problem without assuming any linear growth conditions on the nonlinearities. A new periodic recursive leastsquares (PRLS) algorithm is developed by using nonlinear data weighting. In the sequel, a new periodic adaptive control is proposed to overcome the sector-bounded restriction. Using the Lyapunov technology, the asymptotic convergence and global stability of the proposed periodic adaptive control are shown without requiring linear growth condition. This work is an extended version of the conference paper [23] and a more general case with multiple time-varying parameters is explored and discussed. Simulation study shows the applicability and effectiveness of the proposed approach.

The remainder of this paper is organized as follows. Section 2 gives the problem formulation and the controller design with rigorous convergence analysis. Section 3 extends the result to more general cases with multiple time-varying parameters and time-varying input gains. Some simulation results are provided in Section 4. Finally, some conclusions are given in Section 5.

\section{Problem Formulation and Controller Design}

2.1. Problem Formulation. Consider a discrete-time system with one unknown time-varying parameter

$$
x(t+1)=\theta(t) \xi(x(t))+u(t)
$$

where $x(t) \in R$ is the measurable system state; $u(t) \in R$ is the system control input; $\theta(t) \in R$ is an unknown time-varying parameter with a known periodicity $N>1$, that is, $\theta(t)=$ $\theta(t-N)$; and $\xi(x(t))$ is a known nonlinear scalar function which is bounded for bounded $x(t)$.

It is required that the state, $x(t)$, follow a given reference trajectory $r(t)$. For the simplicity, we use $\xi(t)$ denoting $\xi(x(t))$ in the following discussion.

2.2. Data-Weighting Periodic Adaptation. Defining the tracking error as $e(t)=r(t)-x(t)$, we have

$e(t+1)=x(t+1)-r(t+1)=\theta(t) \xi(t)+u(t)-r(t+1)$.

The new adaptive control mechanism is constructed as follows: where $\widehat{\theta}(t)$ is used to learn the periodic time-varying parameter $\theta(t)$ and updated as follows:

$$
\begin{gathered}
\hat{\theta}(t)= \begin{cases}\hat{\theta}(t-N)-\alpha(t) P(t) \\
\times \xi(t-N) e(t-N+1), & t \in[N, \infty), \\
\widehat{\theta}_{0}, & t \in[0, N),\end{cases} \\
P(t)= \begin{cases}P(t-N) & (4) \\
-\frac{\alpha(t) P^{2}(t-N) \xi^{2}(t-N)}{1+\alpha(t) P(t-N) \xi^{2}(t-N)}, & t \in[N, \infty), \\
P_{0}>0, & t \in[0, N),\end{cases}
\end{gathered}
$$

where the initial value $\widehat{\theta}_{0}$ can be chosen according to some prior knowledge, or simple zero if no prior knowledge is available. Similarly, we can choose $P_{0}$ to be a sufficient large constant over the interval $[0, N) \cdot \alpha(t)=1+\xi^{2}(t)$ is a nonnegative nonlinear data-weighting coefficient.

Remark 1. Here the weighting coefficient $\alpha(t)$ is used to attach more weight to those terms which are more affected by the nonlinearities. For this purpose, we allow $\alpha(t)$ to be a positive nonlinear function of all measured variables up to and including the time instant $t$.

Remark 2. Note that the adaptation process starts only after the first cycle is completed or $t \geq N$. The estimate $\widehat{\theta}(t)$ for $t<N$ is set to be $\widehat{\theta}_{0}$.

For the restriction of the next analysis, an assumption is exposed as follows.

Assumption 3. The unknown time-varying parameters $\theta(t)$ and the target trajectory $r(t)$ are uniformly bounded for all $t$. Without loss of generality, we assume that $\sup _{t}|\theta(t)| \leq \theta_{\max }$, and $\sup _{t}|r(t)| \leq r_{\max }$, where $\theta_{\max }$ and $r_{\max }$ are some positive bounded constants.

Remark 4. Note that, in Assumption 3, we only assume the existence of such bounds, without requiring the exact values.

\subsection{Convergence Analysis}

Theorem 5. For system (1) under Assumption 3, the presented periodic adaptive control algorithm (3)-(5) can guarantee that (a) the parameter estimation value, $\widehat{\theta}(t)$, is bounded for all time $t$ and that $(b)$ the tracking error converges to zero asymptotically.

Proof. There are two parts in the proof of Theorem 5, as shown in the following details.

Part (i): The Boundedness of $\hat{\theta}(t)$. Define the parametric estimation error $\widetilde{\theta}(t)=\theta(t)-\widehat{\theta}(t)$. Substituting the control law (3) into the error dynamics (2) yields

$$
e(t+1)=\tilde{\theta}(t) \xi(t) .
$$


Subtracting $\theta(t)$ from both sides of (4), we have that, for any $t \geq N$,

$$
\widetilde{\theta}(t)=\tilde{\theta}(t-N)-\alpha(t) P(t) \xi(t-N) e(t-N+1) .
$$

Define a nonnegative function $V(t)=P^{-1}(t) \widetilde{\theta}^{2}(t)$, and its difference with respect to the interval $N$ for any $t \geq N$ is

$$
\begin{aligned}
\Delta V(t) & \\
= & V(t)-V(t-N) \\
= & P^{-1}(t) \widetilde{\theta}^{2}(t)-P^{-1}(t-N) \widetilde{\theta}^{2}(t-N) \\
= & P^{-1}(t)[\widetilde{\theta}(t-N)-\alpha(t) P(t) \xi(t-N) e(t-N+1)]^{2} \\
& -P^{-1}(t-N) \widetilde{\theta}^{2}(t-N) \\
= & {\left[P^{-1}(t)-P^{-1}(t-N)\right] \widetilde{\theta}^{2}(t-N) } \\
& -2 \alpha(t) \widetilde{\theta}(t-N) \xi(t-N) e(t-N+1) \\
& +\alpha^{2}(t) P(t) \xi^{2}(t-N) e^{2}(t-N+1) .
\end{aligned}
$$

From (5), it is easy to derive

$$
P^{-1}(t)=P^{-1}(t-N)+\alpha(t) \xi^{2}(t-N) .
$$

Using (9) and the error dynamics (6) leads to

$$
\begin{aligned}
& {\left[P^{-1}(t)-P^{-1}(t-N)\right] \widetilde{\theta}^{2}(t-N)} \\
& \quad=\alpha(t) \xi^{2}(t-N) \widetilde{\theta}^{2}(t-N)=\alpha(t) e^{2}(t-N+1), \\
& -2 \alpha(t) \tilde{\theta}(t-N) \xi(t-T) e(t-N+1) \\
& \quad=-2 \alpha(t) e^{2}(t-N+1) .
\end{aligned}
$$

Substituting (10) and (11) into (8) yields

$$
\Delta V(t)=-\alpha(t) e^{2}(t-N+1)\left(1-\alpha(t) P(t) \xi^{2}(t-N)\right) .
$$

In terms of (5), we can derive

$$
\begin{aligned}
1-\alpha(t) P(t) \xi^{2}(t-N) & =1-\frac{\alpha(t) P(t-N) \xi^{2}(t-N)}{1+\alpha(t) P(t-N) \xi^{2}(t-N)} \\
& =\frac{1}{1+\alpha(t) P(t-N) \xi^{2}(t-N)},
\end{aligned}
$$

so (12) can be rewritten as

$$
\Delta V(t)=-\frac{\alpha(t) e^{2}(t-N+1)}{1+\alpha(t) P(t-N) \xi^{2}(t-N)} \leq 0 .
$$

Thus $V(t)$ is nonincreasing, implying that $\tilde{\theta}(t)$ is bounded. According to Assumption 3, $\theta(t)$ is bounded, which directly leads to the boundedness of $\widehat{\theta}(t)$.
Part (ii): The Convergence of Tracking Error. Applying (14) repeatedly for any $t \in[p N,(p+1) N]$ and noticing $t_{0}=t-p N$, we have

$V(t)$

$$
\begin{aligned}
= & V\left(t_{0}\right) \\
& -\sum_{i=1}^{p} \frac{\alpha\left(t_{0}+i N\right) e^{2}\left(t_{0}+(i-1) N+1\right)}{1+\alpha\left(t_{0}+i N\right) P\left(t_{0}+(i-1) N\right) \xi^{2}\left(t_{0}+(i-1) N\right)} .
\end{aligned}
$$

Since $t_{0} \in[0, N]$, and $p=\left(t-t_{0}\right) / N \rightarrow \infty$, when $t \rightarrow \infty$, according to (15), one can derive that

$$
\begin{aligned}
& \lim _{p \rightarrow \infty} V(t) \\
& =\max _{t_{0} \in[0, N)} V\left(t_{0}\right) \\
& -\lim _{p \rightarrow \infty} \sum_{i=1}^{p}\left(\left(\alpha\left(t_{0}+i N\right) e^{2}\left(t_{0}+(i-1) N+1\right)\right)\right. \\
& \times\left(1+\alpha\left(t_{0}+i N\right) P\left(t_{0}+(i-1) N\right)\right. \\
& \left.\left.\times \xi^{2}\left(t_{0}+(i-1) N\right)\right)^{-1}\right) .
\end{aligned}
$$

Since $V(t)$ is nonnegative and $V\left(t_{0}\right)$ is finite in the interval of $[0, N)$, according to the convergence theorem of the sum of series, we have

$$
\lim _{t \rightarrow \infty} \frac{\alpha(t) e^{2}(t-N+1)}{1+\alpha(t) P(t-N) \xi^{2}(t-N)}=0
$$

or

$$
\lim _{t \rightarrow \infty} \frac{\alpha(t+N) e^{2}(t+1)}{1+\alpha(t+N) P(t) \xi^{2}(t)}=0 .
$$

To show the learning convergence, we need to introduce the following lemma.

Lemma 6. There must exist a constant $d_{0}>0$ such that, for all $t \geq 0$, one has

$$
\frac{P_{0}^{-1}}{\xi^{2}(t)}+\xi^{2}(t-N)+\frac{\xi^{2}(t-N)}{\xi^{2}(t)}>\frac{1}{d_{0}} .
$$

Remark 7. It is worth noting that we only need the existence of $d_{0}$, without requiring its exact value. The proof of Lemma 6 is shown in Appendix A.

According to (9) and Lemma 6, one can derive that

$$
\begin{aligned}
\frac{P^{-1}(t)}{\xi^{2}(t)} & =\frac{P^{-1}(t-N)+\alpha(t) \xi^{2}(t-N)}{\xi^{2}(t)} \\
& \geq \frac{P_{0}^{-1}+\left(1+\xi^{2}(t)\right) \xi^{2}(t-N)}{\xi^{2}(t)} \\
& =\frac{P_{0}^{-1}}{\xi^{2}(t)}+\xi^{2}(t-N)+\frac{\xi^{2}(t-N)}{\xi^{2}(t)}>\frac{1}{d_{0}} ;
\end{aligned}
$$


that is, $P(t) \xi^{2}(t)<d_{0}$. So we have

$$
\begin{aligned}
\frac{1+\alpha(t+N) P(t) \xi^{2}(t)}{\alpha(t+N)} & =\frac{1}{\alpha(t+N)}+P(t) \xi^{2}(t) \\
& <\frac{1}{\alpha(t+N)}+d_{0}<1+d_{0} .
\end{aligned}
$$

Hence,

$$
\begin{aligned}
e^{2}(t+1) & =\frac{1+\alpha(t+N) P(t) \xi^{2}(t)}{\alpha(t+N)} \frac{\alpha(t+N) e^{2}(t+1)}{1+\alpha(t+N) P(t) \xi^{2}(t)} \\
& \leq\left(1+d_{0}\right) \frac{\alpha(t+N) e^{2}(t+1)}{1+\alpha(t+N) P(t) \xi^{2}(t)} .
\end{aligned}
$$

Clearly, we have

$$
\begin{aligned}
0 & \leq \lim _{t \rightarrow \infty} e^{2}(t+1) \\
& \leq\left(1+d_{0}\right) \lim _{t \rightarrow \infty} \frac{\alpha(t+N) e^{2}(t+1)}{1+\alpha(t+N) P(t) \xi^{2}(t)}=0 .
\end{aligned}
$$

In the sequel, one can directly conclude that $\lim _{t \rightarrow \infty} e(t+1)=$ 0 .

\section{Extension to Multiple Parameters and Time-Varying Input Gain}

3.1. Problem Formulation. Consider a scalar system with a specified relative degree $d>1$,

$$
x(t+d)=\left(\boldsymbol{\theta}^{0}(t)\right)^{T} \xi^{0}(t)+b(t) u(t)
$$

where $\boldsymbol{\theta}^{0}=\left[\theta_{1}^{0}, \ldots, \theta_{m}^{0}\right]^{T} \in R^{m}$ are unknown periodic parameters and $\xi^{0}(t)=\xi^{0}(x(t))=\left[\xi_{1}^{0}(x(t)), \ldots, \xi_{m}^{0}(x(t))\right]^{T} \epsilon$ $R^{m}$ is a known vector-valued function. $b(t) \in C[0, \infty)$ is a time-varying and uncertain gain of the system input. The prior information with regard to $b(t)$ is that the control direction is known and invariant; that is, $b(t)$ is either positive or negative and nonsingular for all $t$. Without loss of generality, assume that $b(t) \geq b_{\min }$, where $b_{\min }>0$ is a known lower bound. Note that each unknown parameter $\theta_{i}^{0}(t)$ or $b(t)$ may have its own period $N_{i}$ or $N_{b}$. The periodic adaptive control will still be applicable if there exists a common period $N$, such that $N_{i}$ and $N_{b}$ can divide $N$ with an integer quotient. In such a case, $N$ can be used as the updating period. The presence of uncertain system input gain makes the controller design more complex.

Note that (24) can be incorporated as

$$
x(t+d)=\boldsymbol{\theta}^{T}(t) \boldsymbol{\xi}(t),
$$

where $\boldsymbol{\theta}(t)={ }_{T}\left[\left(\boldsymbol{\theta}^{0}(t)\right)^{T}, b(t)\right]^{T}$ and $\boldsymbol{\xi}(t)=\boldsymbol{\xi}(x(t), u(t))=$ $\left[\left(\xi^{0}(t)\right)^{T}, u(t)\right]^{T}$.

Suppose that a bounded signal $r(t)$ represents the desired output of the system, and the value $r(t+d)$ is known to the controller at time $t$. The objective of periodic adaptive control is to generate a bounded control signal $u(t)$ such that the state $x(t+d)$ asymptotically approaches the specified bounded signal $r(t+d)$.

3.2. Nonlinear Data-Weighting Periodic Adaptation. Defining the tracking error as $e(t)=x(t)-r(t)$, we have

$$
\begin{aligned}
e(t+d) & =x(t+d)-r(t+d)=\boldsymbol{\theta}^{T}(t) \xi(t)-r(t+d) \\
& =\left(\boldsymbol{\theta}^{0}(t)\right)^{T} \xi^{0}(t)+b(t) u(t)-r(t+d) .
\end{aligned}
$$

The periodic adaptive control law is designed as

$$
u(t)=\widehat{b}^{-1}(t)\left[r(t+d)-\left(\widehat{\boldsymbol{\theta}}^{0}(t)\right)^{T} \boldsymbol{\xi}^{0}(t)\right] .
$$

Note that the computation of $u(t)$ requires the inverse of the system input gain estimate $\widehat{b}(t)$ and may cause a singularity in the solution if $\widehat{b}(t)$ is zero. To prevent the input singularity, a semisaturator is applied to the input gain estimation. The parameter updating law is

$$
\begin{aligned}
& \widehat{\boldsymbol{\theta}}(t)=\left\{\begin{array}{cc}
L[\widehat{\boldsymbol{\theta}}(t-N)+\alpha(t) P(t) & \\
\quad \times \boldsymbol{\xi}(t-N) e(t-N+d)], & t \in[N, \infty), \\
L\left[\widehat{\boldsymbol{\theta}}_{0}\right], & t \in[0, N),
\end{array}\right. \\
& P(t)=\left\{\begin{array}{l}
P(t-N) \\
-\frac{\alpha(t) P(t-N) \xi^{T}(t-N) \xi(t-N) P(t-N)}{1+\alpha(t) \xi^{T}(t-N) P(t-N) \xi(t-N)}, \\
t \in[N, \infty), \\
P_{0}, \quad t \in[0, N),
\end{array}\right.
\end{aligned}
$$

where the covariance $P(t)$ is a positive definite matrix of dimension $m+1$ and derived from the relationship $P^{-1}(t)=$ $P^{-1}(t-N)+\xi(t-N) \xi^{T}(t-N)$ by means of the matrix inversion lemma [10]. The initial values of $\widehat{\boldsymbol{\theta}}\left(t_{0}\right), t_{0} \in\{0, \ldots, N\}$, can be chosen arbitrarily provided that $\widehat{b}\left(t_{0}\right) \geq b_{\min }$. Similarly, we can choose the initial values $P\left(t_{0}\right)=P_{0}, \forall t_{0} \in\{0, \ldots, N\}$, with $P_{0}$ being a positive definite matrix. $\alpha(t)=1+\xi^{T}(t) \xi(t)$ is a nonnegative nonlinear data-weighting coefficient.

Let $\mathbf{a}=\left[\mathbf{a}_{1}^{T}, a_{2}\right]$ denote the vector $\widehat{\boldsymbol{\theta}}(t-N)+\alpha(t) P(t) \boldsymbol{\xi}(t-$ $N) e(t-N+d)$; the semisaturator is defined as

$$
L[\mathbf{a}]= \begin{cases}{\left[\mathbf{a}_{1}^{T}, a_{2}\right]^{T},} & a_{2}>b_{\min } \\ {\left[\mathbf{a}_{1}^{T}, b_{\min }\right]^{T},} & a_{2} \leq b_{\min }\end{cases}
$$

3.3. Convergence Analysis. An assumption is introduced as follows.

Assumption 8. The unknown time-varying parameters $\boldsymbol{\theta}(t)$ and the target trajectory $r(t)$ are uniformly bounded for all $t$. Without loss of generality, we assume that $\sup _{t}\|\boldsymbol{\theta}(t)\| \leq \theta_{\max }$, and $\sup _{t}|r(t)| \leq r_{\max }$, where $\theta_{\max }$ and $r_{\max }$ are some bounded constants.

The validity of the above periodic adaption law is verified by the following theorem. 
Theorem 9. For system (24) under Assumption 8, the proposed period adaptive control scheme (27)-(30) has the following properties.

(a) The parameter estimation error is bounded; that is, $\|\widehat{\boldsymbol{\theta}}(t)-\boldsymbol{\theta}(t)\|^{2} \leq \kappa_{1}\left\|\widehat{\boldsymbol{\theta}}\left(t_{0}\right)-\boldsymbol{\theta}\left(t_{0}\right)\right\|^{2}$, where $\kappa_{1}=$ $\left(\lambda_{\max }\left[P\left(t_{0}\right)^{-1}\right]\right) /\left(\lambda_{\text {min }}\left[P\left(t_{0}\right)^{-1}\right]\right), t_{0} \in\{0, \ldots, N\}$.

(b) The tracking error converges to zero asymptotically as time instant t approaches to infinity.

Proof. According to (27),

$$
r(t+d)=\left(\widehat{\boldsymbol{\theta}}^{0}(t)\right)^{T} \xi^{0}(t)+\widehat{b}(t) u(t)=\widehat{\boldsymbol{\theta}}^{T}(t) \boldsymbol{\xi}(t) .
$$

Substituting (31) into (26), we have

$$
\begin{aligned}
e(t+d) & =\boldsymbol{\theta}^{T}(t) \boldsymbol{\xi}(t)-r(t+d) \\
& =\boldsymbol{\theta}^{T}(t) \boldsymbol{\xi}(t)-\widehat{\boldsymbol{\theta}}^{T}(t) \boldsymbol{\xi}(t)=\widetilde{\boldsymbol{\theta}}^{T}(t) \boldsymbol{\xi}(t),
\end{aligned}
$$

where $\widetilde{\boldsymbol{\theta}}(t)=\boldsymbol{\theta}(t)-\widehat{\boldsymbol{\theta}}(t)=\boldsymbol{\theta}(t)-L[\mathbf{a}]$.

Define a nonnegative function $V(t)=\widetilde{\boldsymbol{\theta}}^{T}(t) P^{-1}(t) \widetilde{\boldsymbol{\theta}}(t)$, and its difference with respect to the interval $N$ for any $t \geq N$ is

$$
\begin{aligned}
\Delta V(t)= & V(t)-V(t-N) \\
= & \widetilde{\boldsymbol{\theta}}^{T}(t) P^{-1}(t) \tilde{\boldsymbol{\theta}}(t)-\tilde{\boldsymbol{\theta}}^{T}(t-N) P^{-1}(t-N) \tilde{\boldsymbol{\theta}}(t-N) \\
= & (\boldsymbol{\theta}(t)-L[\mathbf{a}])^{T} P^{-1}(t)(\boldsymbol{\theta}(t)-L[\mathbf{a}]) \\
& -\widetilde{\boldsymbol{\theta}}^{T}(t-N) P^{-1}(t-N) \tilde{\boldsymbol{\theta}}(t-N) .
\end{aligned}
$$

Note that, when $\widehat{b}(t) \geq b_{\min }, L[\widehat{b}(t)]=\widehat{b}(t)$ and $\mid b(t)-$ $L[\widehat{b}(t)]|=| b(t)-\widehat{b}(t) \mid$. When $\widehat{b}(t)<b_{\min }, L[\widehat{b}(t)]=b_{\min }$ and the relationship $|b(t)-L[\widehat{b}(t)]|=\left|b(t)-b_{\min }\right|<|b(t)-\widehat{b}(t)|$ holds. Thus the magnitude of the estimation error is the same or larger if no saturator is applied. As a result, we have

$$
(\boldsymbol{\theta}(t)-L[\mathbf{a}])^{T}(\boldsymbol{\theta}(t)-L[\mathbf{a}]) \leq(\boldsymbol{\theta}(t)-\mathbf{a})^{T}(\boldsymbol{\theta}(t)-\mathbf{a}) .
$$

Furthermore, for a positive definite matrix $P^{-1}(t)$, the following also holds:

$$
\begin{aligned}
(\boldsymbol{\theta}(t) & -L[\mathbf{a}])^{T} P^{-1}(t)(\boldsymbol{\theta}(t)-L[\mathbf{a}]) \\
& \leq(\boldsymbol{\theta}(t)-\mathbf{a})^{T} P^{-1}(t)(\boldsymbol{\theta}(t)-\mathbf{a}) .
\end{aligned}
$$

Thus, we can further simplify (33) as

$\Delta V(t)$

$$
\begin{aligned}
\leq & (\boldsymbol{\theta}(t)-\mathbf{a})^{T} P^{-1}(t)(\boldsymbol{\theta}(t)-\mathbf{a}) \\
& -\widetilde{\boldsymbol{\theta}}^{T}(t-N) P^{-1}(t-N) \widetilde{\boldsymbol{\theta}}(t-N) \\
= & {[\widetilde{\boldsymbol{\theta}}(t-N)-\alpha(t) P(t) \boldsymbol{\xi}(t-N) e(t-N+d)]^{T} } \\
& \times P^{-1}(t)[\widetilde{\boldsymbol{\theta}}(t-N)-\alpha(t) P(t) \boldsymbol{\xi}(t-N) e(t-N+d)] \\
& -\widetilde{\boldsymbol{\theta}}^{T}(t-N) P^{-1}(t-N) \widetilde{\boldsymbol{\theta}}(t-N) \\
= & \widetilde{\boldsymbol{\theta}}^{T}(t-N)\left(P^{-1}(t)-P^{-1}(t-N)\right) \tilde{\boldsymbol{\theta}}(t-N) \\
& -2 \alpha(t) \widetilde{\boldsymbol{\theta}}^{T}(t-N) \boldsymbol{\xi}(t-N) e(t-N+d) \\
& +\alpha^{2}(t) \boldsymbol{\xi}^{T}(t-N) P(t) \boldsymbol{\xi}(t-N) e^{2}(t-N+d) .
\end{aligned}
$$

From the matrix inversion lemma [10] and (29), we have

$$
P^{-1}(t)=P^{-1}(t-N)+\alpha(t) \xi(t-N) \xi^{T}(t-N) .
$$

Thus we can rearrange (36) as

$\Delta V(t)$

$$
\begin{aligned}
\leq & \alpha(t) \widetilde{\boldsymbol{\theta}}^{T}(t-N) \boldsymbol{\xi}^{T}(t-N) \boldsymbol{\xi}(t-N) \tilde{\boldsymbol{\theta}}(t-N) \\
& -2 \alpha(t) \widetilde{\boldsymbol{\theta}}^{T}(t-N) \boldsymbol{\xi}(t-N) e(t-N+d) \\
& +\alpha^{2}(t) \boldsymbol{\xi}^{T}(t-N) P(t) \boldsymbol{\xi}(t-N) e^{2}(t-N+d) .
\end{aligned}
$$

Using the error dynamics (32), we can simplify (38) as

$$
\begin{aligned}
\Delta V(t) \leq & \alpha(t) e^{2}(t-N+d)-2 \alpha(t) e^{2}(t-N+d) \\
& +\alpha^{2}(t) \xi^{T}(t-N) P^{-1}(t) \xi(t-N) e^{2}(t-N+d) \\
= & -\alpha(t)\left(1-\alpha(t) \xi^{T}(t-N) P(t) \xi(t-N)\right) \\
& \times e^{2}(t-N+d) .
\end{aligned}
$$

In order to evaluate the relationship between $V(t)$ and $V(t-N)$ described by (39), look at the term $1-\alpha(t) \xi^{T}(t-$ $N) P(t) \boldsymbol{\xi}(t-N)$ in (39).

From (29), one can derive

$$
\begin{aligned}
\alpha(t) & \xi^{T}(t-N) P(t) \xi(t-N) \\
= & \alpha(t) \xi^{T}(t-N) P(t-N) \xi(t-N) \\
& -\frac{\alpha^{2}(t)\left[\xi^{T}(t-N) P(t-N) \xi(t-N)\right]^{2}}{1+\alpha(t) \xi^{T}(t-N) P(t-N) \xi(t-N)} \\
= & \frac{\alpha(t) \xi^{T}(t-N) P(t-N) \xi^{T}(t-N)}{1+\alpha(t) \xi^{T}(t-N) P(t-N) \xi(t-N)} .
\end{aligned}
$$


Therefore,

$$
\begin{aligned}
1- & \alpha(t) \xi^{T}(t-N) P(t) \xi(t-N) \\
& =1-\frac{\alpha(t) \xi^{T}(t-N) P(t-N) \xi^{T}(t-N)}{1+\alpha(t) \xi^{T}(t-N) P(t-N) \xi(t-N)} \\
& =\frac{1}{1+\alpha(t) \xi^{T}(t-N) P(t-N) \xi(t-N)} .
\end{aligned}
$$

Note that $P(t)$ is a positive definite matrix for any $t$; we can immediately obtain that

$$
\Delta V(t) \leq-\frac{\alpha(t) e^{2}(t-N+d)}{1+\alpha(t) \xi^{T}(t-N) P(t-N) \xi(t-N)} \leq 0 .
$$

Thus $V(t)$ is nonincreasing, and for any $t \in[p N,(p+1) N]$, $t_{0}=t-p N$, we have

$$
\begin{aligned}
\tilde{\boldsymbol{\theta}}^{T}(t) P^{-1}(t) \tilde{\boldsymbol{\theta}}(t) & \leq \tilde{\boldsymbol{\theta}}^{T}(t-N) P^{-1}(t-N) \tilde{\boldsymbol{\theta}}(t-N) \\
& \leq \cdots \leq \tilde{\boldsymbol{\theta}}^{T}\left(t_{0}\right) P^{-1}\left(t_{0}\right) \tilde{\boldsymbol{\theta}}\left(t_{0}\right) .
\end{aligned}
$$

From (37), we can derive

$$
\lambda_{\min }\left[P(t)^{-1}\right] \geq \lambda_{\min }\left[P(t-N)^{-1}\right] \geq \cdots \geq \lambda_{\min }\left[P\left(t_{0}\right)^{-1}\right] .
$$

Equation (44) implies that

$$
\begin{aligned}
& \lambda_{\min } {\left[P\left(t_{0}\right)^{-1}\right]\|\widetilde{\boldsymbol{\theta}}(t)\|^{2} } \\
& \leq \lambda_{\min }\left[P(t)^{-1}\right]\|\widetilde{\boldsymbol{\theta}}(t)\|^{2} \leq \widetilde{\boldsymbol{\theta}}(t)^{T}\left[P(t)^{-1}\right] \widetilde{\boldsymbol{\theta}}(t) \\
& \leq \widetilde{\boldsymbol{\theta}}\left(t_{0}\right)^{T}\left[P\left(t_{0}\right)^{-1}\right] \widetilde{\boldsymbol{\theta}}\left(t_{0}\right) \leq \lambda_{\max }\left[P\left(t_{0}\right)^{-1}\right]\left\|\widetilde{\boldsymbol{\theta}}\left(t_{0}\right)\right\|^{2},
\end{aligned}
$$

which establishes conclusion (a) of Theorem 9; that is,

$$
\|\widehat{\boldsymbol{\theta}}(t)-\boldsymbol{\theta}(t)\|^{2} \leq \kappa_{1}\left\|\widehat{\boldsymbol{\theta}}\left(t_{0}\right)-\boldsymbol{\theta}\left(t_{0}\right)\right\|^{2} .
$$

Following the same steps that lead to (18) in Theorem 5 and by virtue of (42), one can conclude that

$$
\lim _{t \rightarrow \infty} \frac{\alpha(t) e^{2}(t-N+d)}{1+\alpha(t) \xi^{T}(t-N) P(t-N) \xi(t-N)}=0 .
$$

Since the nonlinear function is not sector-bounded, the following lemma is introduced to show the convergence performance.

Lemma 10. There must exist a constant $d_{1}>0$ such that, for all $t \geq 0$, one has

$$
\begin{aligned}
& \frac{P_{0}^{-1}}{\xi^{T}(t) \xi(t)}+\xi^{T}(t-N) \xi(t-N) \\
& +\frac{\xi^{T}(t-N) \xi(t-N)}{\xi^{T}(t) \xi(t)}>\frac{1}{d_{1}} .
\end{aligned}
$$

Proof. See Appendix B.

Following the same steps that lead to (23) in Theorem 5 and using (48), it is easy to derive that

$$
\begin{aligned}
0 & \leq \lim _{t \rightarrow \infty} e^{2}(t+d) \\
& \leq\left(1+d_{1}\right) \lim _{k \rightarrow \infty}\left\|\frac{\alpha(t+N) e^{2}(t+d)}{1+\alpha(t+N) \xi^{T}(t) P(t) \xi(t)}\right\|=0 .
\end{aligned}
$$

Hence, we can directly conclude $\lim _{t \rightarrow \infty} e(t+d)=0$.

\section{Illustrative Examples}

Consider a system

$$
x(t+1)=\theta(t) \frac{x(t)^{2}+x(t)^{3}+x(t)^{4}}{1+x(t)^{2}}+b(t) u(t),
$$

where $\theta(t)=1+0.5 \sin (t \pi / 10)$ and $b(t)=1+0.5 \cos (t \pi / 10)$. The common periodicity of $\theta(t)$ and $b(t)$ is known as 20 .

Furthermore, we can see that the nonlinear function above is not satisfied with the linear growth condition.

It is required that $x(t)$ track a given reference

$$
r(t)= \begin{cases}0.8 \sin \left(\frac{t \pi}{25}\right)+0.3 \cos \left(\frac{t \pi}{25}\right), & 0 \leq t \leq 30, \\ (-1)^{\operatorname{round}(t / 25)}, & 30<t \leq 70 \\ 2 \sin \left(\frac{t \pi}{30}\right)+3 \cos \left(\frac{t \pi}{20}\right), & 70<t \leq 100 .\end{cases}
$$

Note that the given desired trajectory $r(t)$ is also a period function, but its periodicity known as 100 has nothing to do with the common periodicity of the unknown parameters. In the simulation, the periodicity used to update the period parameters is 20 , instead of 100 .

In the simulation, the initial value $x(0)$ is set as 0 . The other parameters are chosen as $\hat{\theta}_{0}=1.5, b(t)=1.5, P_{0}=$ $\left[\begin{array}{cc}15 & 0 \\ 0 & 15\end{array}\right]$, and $d=2$ over the first period $[0,20]$. By using the presented data-weighting periodic adaptive control (27)(29), the simulation results are shown in Figures 1-3, respectively. Figure 1 is the profile of nonlinear data-weighting factor $\alpha(t)$. Figure 2 is the tracking performance of system output. And the convergence of tracking error is shown in Figure 3, where $e_{\max }(i)=\max _{t \in\{(i-1) N,(i-1) N+1, \ldots, i N\}}|e(t)|$ is used to record the maximum absolute tracking error during the $i$ th period. Obviously, the proposed method results in good convergence in a pointwise manner.

Apparently, the effectiveness of the proposed dataweighting periodic adaptive control can be seen from Figures 1-3. Although the nonlinear system in the simulation is not sector-bounded, the tracking error converges asymptotically to zero as period number approaches to infinity. The reason is that the nonlinearities are compensated by using the data weighting factor $\alpha(t)$ (Figure 1). 


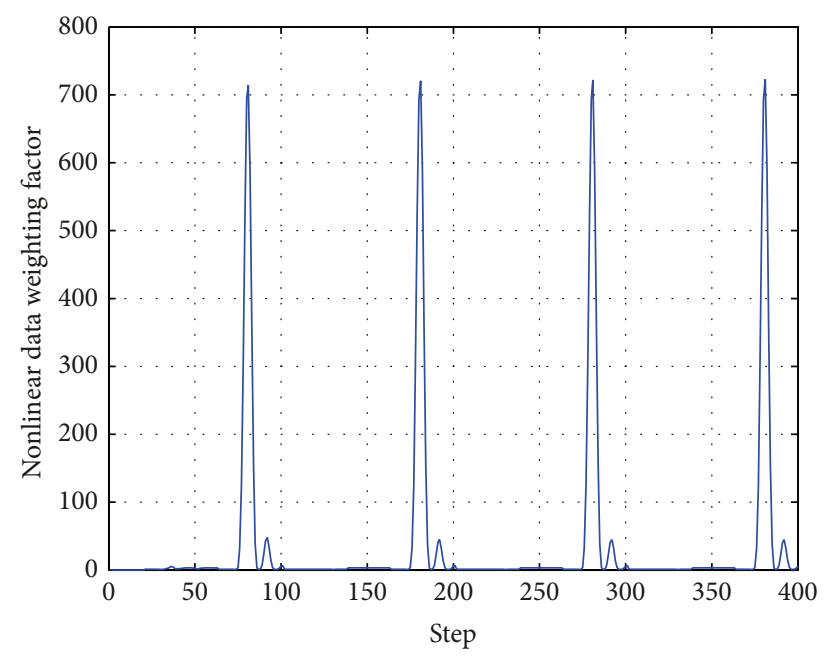

FIGURE 1: The profile of nonlinear data-weighting factor $\alpha(t)$.

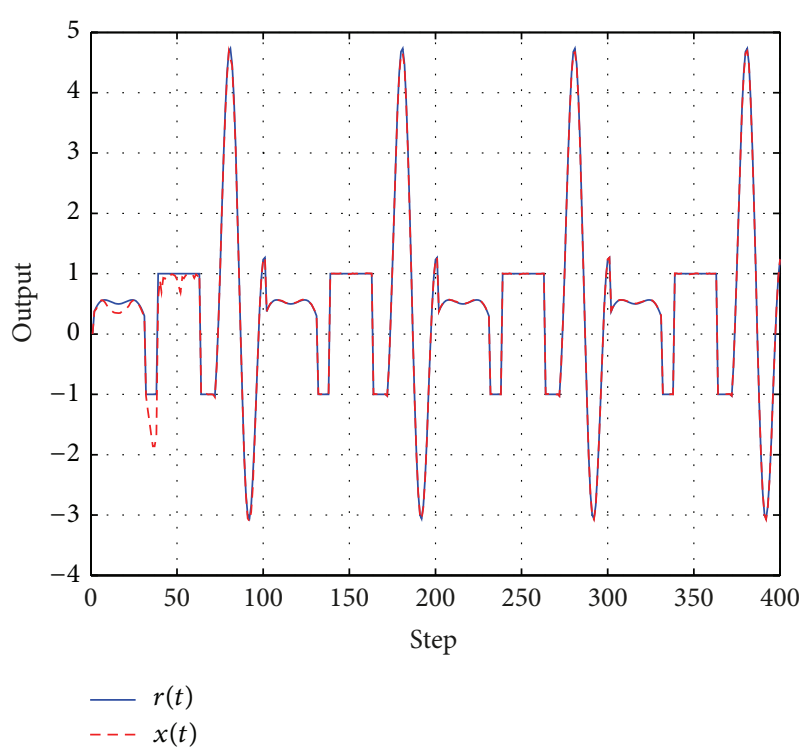

FIGURE 2: The tracking performance of system output.

For comparison, the following standard periodic adaptive control [22] without nonlinear data-weighting factor is applied:

$$
u(t)=\widehat{b}^{-1}(t)\left[r(t+1)-\left(\widehat{\boldsymbol{\theta}}^{0}(t)\right)^{T} \xi^{0}(t)\right]
$$

$$
\begin{aligned}
& \widehat{\boldsymbol{\theta}}(t) \\
& \quad=\left\{\begin{array}{cc}
L[\widehat{\boldsymbol{\theta}}(t-N) & \\
+P(t) \boldsymbol{\xi}(t-N) e(t-N+1)], & t \in[N, \infty), \\
L\left[\widehat{\boldsymbol{\theta}}_{0}\right], & t \in[0, N),
\end{array}\right.
\end{aligned}
$$

$P(t)$

$$
=\left\{\begin{aligned}
P(t-N) & \\
-\frac{P(t-N) \xi^{T}(t-N) \xi(t-N) P(t-N)}{1+\xi^{T}(t-N) P(t-N) \xi(t-N)}, & t \in[N, \infty), \\
& t \in[0, N) .
\end{aligned}\right.
$$

By selecting the same controller parameters and the same initial value $x(0)=0$ as that in the previous simulation, the profile of tracking error is shown in Figure 4. It is obvious that a finite time escape phenomenon may occur for the tracking error without using the nonlinear data-weighting factor $\alpha(t)$.

\section{Conclusion}

A new adaptive control is proposed with periodic leastsquares estimate for a class of discrete-time systems to address periodic time-varying parameters. The only prior knowledge needed in the periodic adaptation is the periodicity. The periodic parameter updating law proposed here is updated in the same instance of two consecutive periods. A major distinct feature is that a nonlinear data-weighting function is introduced into the parameter updating law to address nonlinearities without requiring any growth condition. Both theoretical analysis and numerical simulations verify the effectiveness of the proposed approach.

\section{Appendices}

\section{A. Proof of Lemma 6}

Proof. We arbitrarily choose a positive constant $\delta_{0}$ and examine the following two cases.

Case 1. When $\xi^{2}(t-N)>\delta_{0}$, then (19) is satisfied with $d_{0}>\delta_{0}^{-1}$.

Case 2. When $\xi^{2}(t-N) \leq \delta_{0}$, according to the definition of $V(t)$ and the relationship of (14), it is obvious that

$$
P_{0}^{-1} \widetilde{\theta}^{2}(t) \leq P^{-1}(t) \widetilde{\theta}^{2}(t) \leq P_{0}^{-1} \widetilde{\theta}^{2}\left(t_{0}\right) .
$$

Hence, for all $t \geq 0$, one has

$$
|\widetilde{\theta}(t)| \leq\left|\widetilde{\theta}\left(t_{0}\right)\right| \leq \widetilde{\theta}_{\max },
$$

where $\widetilde{\theta}_{\max }=\max _{t_{0} \in\{0, \ldots, T\}}\left\{\left|\widetilde{\theta}\left(t_{0}\right)\right|\right\}$.

According to (1) and (3), we have

$$
\begin{aligned}
|x(t-N+1)| & =|\widetilde{\theta}(t-N) \xi(t-N)+r(t-N+1)| \\
& \leq|\widetilde{\theta}(t-N)||\xi(t-N)|+|r(t-N+1)| \\
& \leq \widetilde{\theta}_{\max } \sqrt{\delta_{0}}+r_{\max } .
\end{aligned}
$$




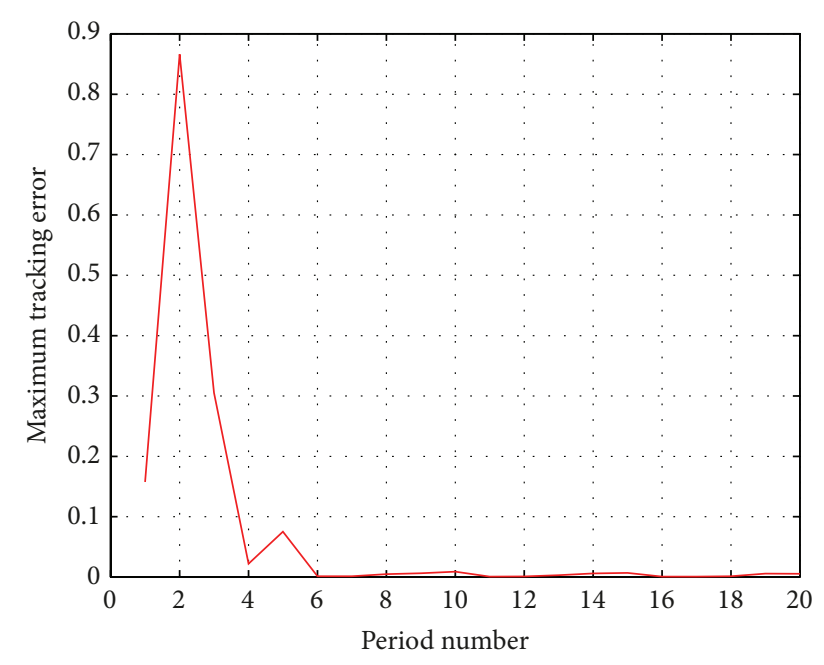

FIGURE 3: The convergence property of tracking error with period.

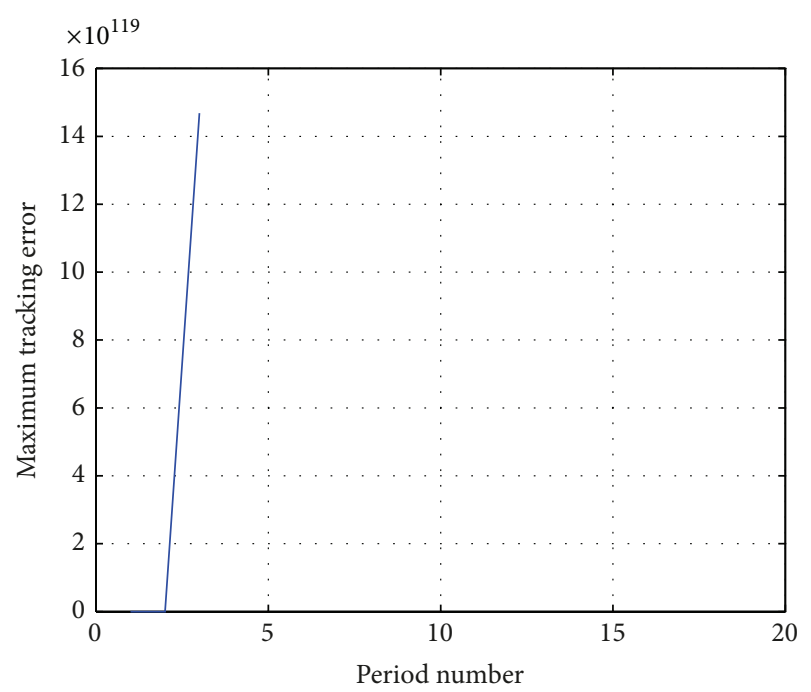

Figure 4: Finite time escape phenomenon of tracking error with standard periodic adaptive control.

Since nonlinear function $\xi(x)$ is bounded for bounded $x$, apparently there exists a constant $\delta_{1}$ such that $\xi^{2}(t-N+1) \leq$ $\delta_{1}$ is bounded.

Apparently, we have

$$
\begin{aligned}
|x(t-N+2)|= & |\widetilde{\theta}(t-N+1) \xi(t-N+1)+r(t-N+2)| \\
\leq & |\widetilde{\theta}(t-N+1)||\xi(t-N+1)| \\
& +|r(t-N+2)| \\
\leq & \tilde{\theta}_{\max } \sqrt{\delta_{1}}+r_{\max }
\end{aligned}
$$

and there exists a constant $\delta_{2}$ such that $\xi^{2}(t-N+2) \leq \delta_{2}$.
By analogy, there exists a constant $\delta_{N-1}$ such that

$$
\begin{aligned}
|x(t)| & =|\widetilde{\theta}(t-1) \xi(t-1)+r(t)| \\
& \leq|\widetilde{\theta}(t-1)||\xi(t-1)|+|r(t)| \\
& \leq \widetilde{\theta}_{\max } \sqrt{\delta_{N-1}}+r_{\max } .
\end{aligned}
$$

Thus we can find a constant $\delta_{N}$, which is bounded since $N$ is a finite periodicity, such that $\xi^{2}(t) \leq \delta_{N}$. Then (19) is satisfied with $d_{0}>\delta_{N} P_{0}$.

The above discussion shows that (19) is satisfied for all $t$ with $d_{0}>\max \left\{\delta_{0}^{-1}, \delta_{N} P_{0}\right\}$.

\section{B. Proof of Lemma 10}

Proof. For any $t \in[p N,(p+1) N]$ and $t_{0}=t-p N$, arbitrarily choose a positive constant $\sigma_{0}$ and examine the following two cases.

$$
\begin{aligned}
& \text { (1) } \boldsymbol{\xi}^{T}(t-N) \boldsymbol{\xi}(t-N)>\sigma_{0} \text {. Then (48) is satisfied with } \\
& d_{1}>\sigma_{0}^{-1} . \\
& \text { (2) } \xi^{T}(t-N) \boldsymbol{\xi}(t-N) \leq \sigma_{0} \text {. Since } \\
& \xi^{T}(t-N) \xi(t-N)=\xi^{0}(t-N)^{T} \xi^{0}(t-N)+b^{2}(t-N)
\end{aligned}
$$

we know that both $\left\|\xi^{0}(t-N)\right\|$ and $b(t-N)$ are bounded.

From (45), we know that for all $t \geq 0$

$$
\|\widetilde{\boldsymbol{\theta}}(t)\| \leq \sqrt{\kappa_{1}}\left\|\widetilde{\boldsymbol{\theta}}\left(t_{0}\right)\right\| \leq \sqrt{\kappa_{1}} \widetilde{\boldsymbol{\theta}}_{\max }
$$

where $\widetilde{\boldsymbol{\theta}}_{\text {max }}=\max _{t_{0} \in\{0, \ldots, T\}}\left\{\left\|\widetilde{\boldsymbol{\theta}}\left(t_{0}\right)\right\|\right\}$.

$\widehat{\boldsymbol{\theta}}^{0}(t)$ and $\widehat{b}(t)$ have been shown bounded; thus

$$
|u(t)| \leq\left|\widehat{b}^{-1}(t)\right|\left[|r(t+d)|+\left\|\hat{\boldsymbol{\theta}}^{0}(t)\right\|\left\|\xi^{0}(t)\right\|\right] \leq q\left\|\xi^{0}(t)\right\|
$$

for some constant $q$. Clearly the control input signal $|u(t)|$ is bounded.

For any $t_{0} \in[-d, N]$, since the selected initial state values $x\left(t_{0}\right)$ are bounded, clearly $\xi^{0}\left(t_{0}\right)$ and thus $u\left(t_{0}\right)$ are bounded according to (B.3).

For the convenience, we denote $\xi_{\max }^{0}=\max _{t_{0} \in\{-d \ldots, N\}}$ $\left\{\left\|\xi^{0}\left(t_{0}\right)\right\|\right\}, u_{\max }=\max _{t_{0} \in\{-d, \ldots, N\}}\left\{\left|u\left(t_{0}\right)\right|\right\}$, and $b_{\max }=$ $\max _{t_{0} \in\{-d, \ldots, N\}}\left\{\left|b\left(t_{0}\right)\right|\right\}$ in the following.

From the system (24), it is easy to get

$$
\begin{aligned}
|x(N+1)| & =|x(-d+N+1+d)| \\
& \leq\left\|\boldsymbol{\theta}^{0}(-d+N+1)\right\|\left\|\xi^{0}(0)\right\|+|b(0)||u(0)| \\
& \leq \widetilde{\boldsymbol{\theta}}_{\text {max }} \xi_{\text {max }}^{0}+b_{\text {max }} u_{\max }=\sigma_{1} .
\end{aligned}
$$

Similarly,

$$
|x(N+2)| \leq \sigma_{2}, \ldots,|x(N+N)| \leq \sigma_{N} .
$$


As a result, $\xi^{0}\left(t_{1}\right)$ is bounded for the bounded $x\left(t_{1}\right)$, with $t_{1} \in[N+1,2 N]$. According to (B.3), $u\left(t_{1}\right)$ is bounded; thus $\left\|\boldsymbol{\xi}\left(t_{1}\right)\right\|$ is bounded too.

By the same steps, one can conclude that $\|\boldsymbol{\xi}(t)\|$ is bounded. Without loss of generality, we assume that $\boldsymbol{\xi}^{T}(t) \boldsymbol{\xi}(t) \leq \gamma_{1}$; then (48) is satisfied with $d_{1}>\gamma_{1} P_{0}$.

The above discussion shows that (48) is satisfied for all $t$ with $d_{1}>\max \left\{\sigma_{0}, \gamma_{1} P_{0}\right\}$.

\section{Conflict of Interests}

The authors declare that there is no conflict of interests regarding the publication of this paper.

\section{Acknowledgment}

This work was supported by National Science Foundation of China (60974040, 61374102, and 61120106009).

\section{References}

[1] T. Inoue, M. Nakano, and S. Iwai, "High accuracy control of servomechanism for repeated contouring," in Proceedings of the 10th Annual Symposium on Incremental Motion Control Systems and Devices, pp. 258-292, 1981.

[2] T. Inoue, M. Nakano, and S. Iwai, "High accuracy control of a proton synchrotron magnet power supply," in Proceedings of the 8th World Congress of the International Federation of Automatic Control (IFAC), pp. 216-221, 1981.

[3] S. Arimoto, Mathematical Theory of Learning with Applications to Robot Control, Adaptive and Learning Systems, K. S. Narendra, Ed., Plenum Publishing, 1986.

[4] S. Hara, Y. Yamamoto, T. Omata, and M. Nakano, "Repetitive control system: a new type servo system for periodic exogenous signals," IEEE Transactions on Automatic Control, vol. 33, no. 7, pp. 659-668, 1988.

[5] Y. Yamamoto and S. Hara, "Relationships between internal and external stability of infinite dime $\mathrm{n}$ sional systems with application s to a servo problem," in Proceedings of the 26th Conference on Decision and Control, pp. 1558-1563, 1987.

[6] N. Sadegh, "Synthesis and stability analysis of repetitive controllers," in Proceedings of the American Control Conference, vol. 3, pp. 2634-2639, June 1991.

[7] M. Tomizuka, T.-C. Tsao, and K.-K. Chew, "Analysis and synthesis of discrete-time repetitive controllers," Journal of Dynamic Systems, Measurement and Control, Transactions of the ASME, vol. 111, no. 3, pp. 353-358, 1989.

[8] J. Hu and M. Tomizuka, "A new plug-in adaptive controller for rejection of periodic disturbances," in Proceedings of the ASME Winter Annual Meeting, vol. 33, DSC, Atlanta, Ga, USA, December 1991.

[9] R. Horowitz, "Learning control of robot manipulators," Journal of Dynamic Systems, Measurement and Control, vol. 115, no. 2, pp. 402-411, 1993.

[10] G. C. Goodwin and K. S. Sin, Adaptive Filtering Prediction and Control, Prentice-Hall, Englewood Cliffs, NJ, USA, 1984.

[11] I. Kanellakopoulos, "A discrete-time adaptive nonlinear system," IEEE Transactions on Automatic Control, vol. 39, no. 11, pp. 2362-2365, 1994.
[12] K. J. Aström and B. Wittenmark, Adaptive Control, AddisonWesley, Reading, Mass, USA, 2nd edition, 1995.

[13] L. L. Xie and L. Guo, "How much uncertainty can be dealt with by feedback?” IEEE Transactions on Automatic Control, vol. 45, no. 12, pp. 2203-2217, 2000.

[14] C. Yang, L. Zhai, S. S. Ge, T. Chai, and H. L. Tong, "Adaptive model reference control of a class of MIMO discrete-time systems with compensation of nonparametric uncertainty," in Proceedings of the American Control Conference (ACC '08), pp. 4111-4116, Seattle, Wash, USA, June 2008.

[15] R. Marino and P. Tomei, "Adaptive control of linear timevarying systems," Automatica, vol. 39, no. 4, pp. 651-659, 2003.

[16] J. Xu, "A new periodic adaptive control approach for timevarying parameters with known periodicity," IEEE Transactions on Automatic Control, vol. 49, no. 4, pp. 579-583, 2004.

[17] Z. Wang, M. M. Polycarpou, J. G. Uber, and F. Shang, "Adaptive control of water quality in water distribution networks," IEEE Transactions on Control Systems Technology, vol. 14, no. 1, pp. 149-156, 2006.

[18] D. Gorinevsky, "Periodic adaptive stabilization of unstable nonminimum phase system," in Proceedings of the American Control Conference, pp. 2995-2999, July 1994.

[19] W. E. Dixon, E. Zergeroglu, D. M. Dawson, and B. T. Costic, "Repetitive learning control: a Lyapunov-based approach," IEEE Transactions on Systems, Man, and Cybernetics B: Cybernetics, vol. 32, no. 4, pp. 538-545, 2002.

[20] M. Sun and S. S. Ge, "Adaptive repetitive control for a class of nonlinearly parametrized systems," IEEE Transactions on Automatic Control, vol. 51, no. 10, pp. 1684-1688, 2006.

[21] K. Abidi and J. X. Xu, "A discrete-time periodic adaptive control approach for time-varying parameters with known periodicity," IEEE Transactions on Automatic Control, vol. 53, no. 2, pp. 575581, 2008.

[22] R. Chi, J. Li, Z. Hou, and S. Jin, "Periodic adaptive identification and control for a class of discrete time-varying systems," in Proceedings of the 23th Chinese Control and Decision Conference (CCDC '11), pp. 155-159, Mianyang, China, May 2011.

[23] R. Chi, Y. Zhang, S. Jin, and Z. Hou, "A discrete-time periodic adaptive control for systems in the presence of nonsector nonlinearities," in Proceedings of the 30th Chinese Control Conference (CCC '11), pp. 2419-2422, Yantai, China, July 2011.

[24] J. X. Xu, "A survey on iterative learning control for nonlinear systems," International Journal of Control, vol. 84, no. 7, pp. 1275-1294, 2011.

[25] L. G. Sinson and E. K. P. Chong, "Non-reset iterative learning control," in Proceedings of the 35th Conference on Decision and Control, pp. 3062-3063, Kobe, Japan, December 1996.

[26] K. L. Moore, "A non-standard iterative learning control approach to tracking periodic signals in discrete-time nonlinear systems," International Journal of Control, vol. 73, no. 10, pp. 955-967, 2000.

[27] R. H. Chi, Z. S. Hou, and J.-X. Xu, "Adaptive ILC for a class of discrete-time systems with iteration-varying trajectory and random initial condition," Automatica, vol. 44, no. 8, pp. 22072213, 2008

[28] R. Chi, Z. Hou, and S. Jin, "Data-weighting based discretetime adaptive iterative learning control for nonsector nonlinear systems with iteration-varying trajectory and random initial condition," Journal of Dynamic Systems, Measurement and Control, Transactions of the ASME, vol. 134, no. 2, Article ID 021016, 2012. 


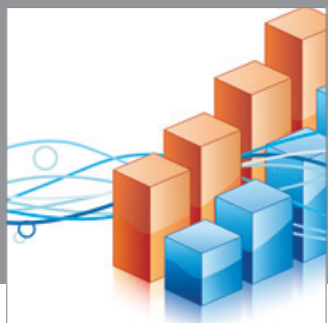

Advances in

Operations Research

mansans

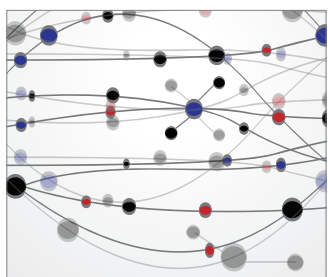

The Scientific World Journal
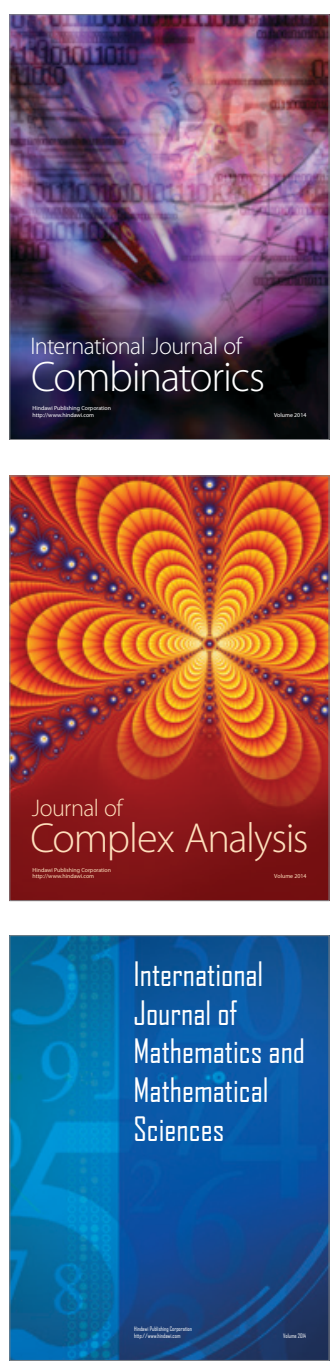
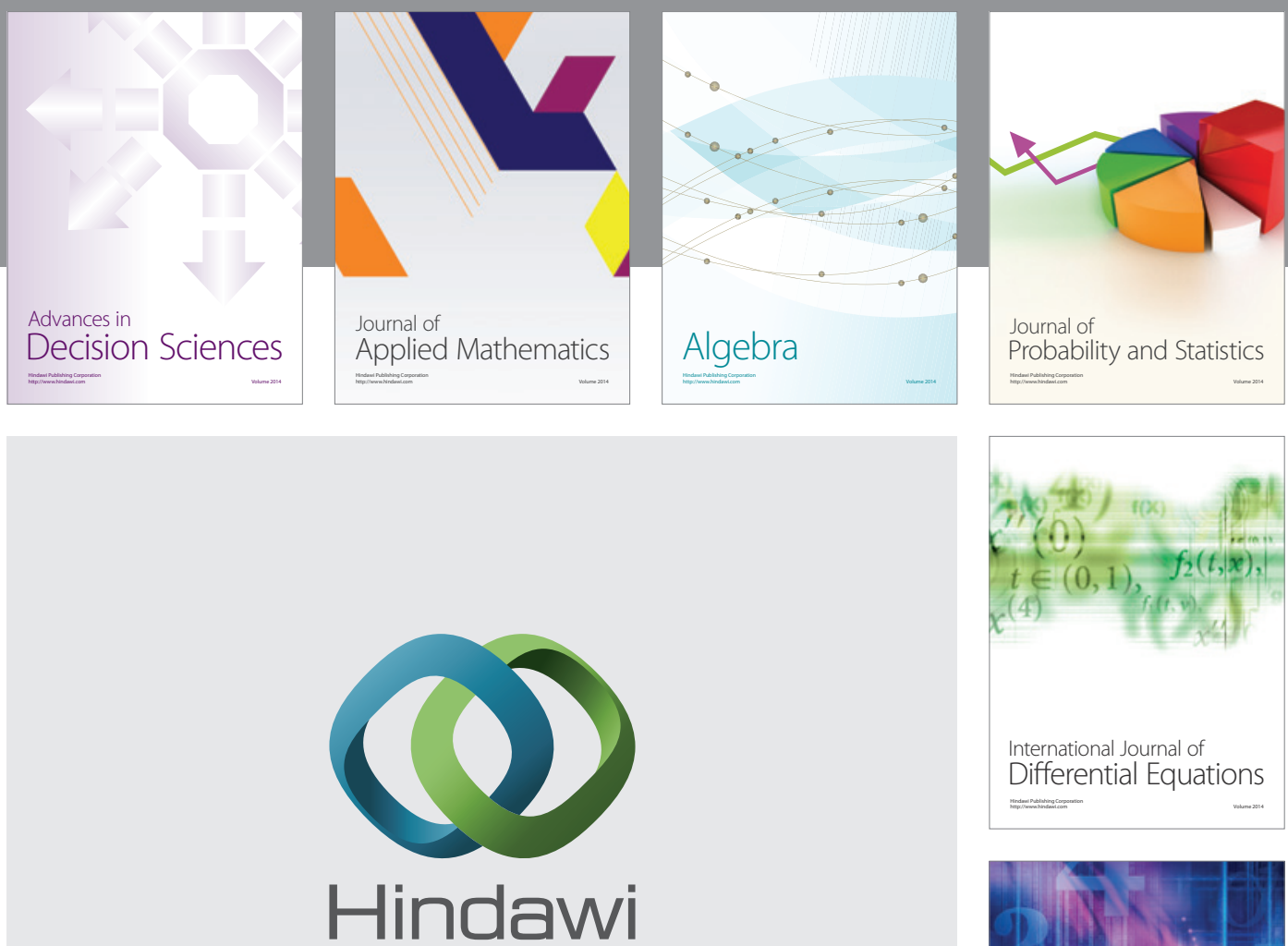

Submit your manuscripts at http://www.hindawi.com
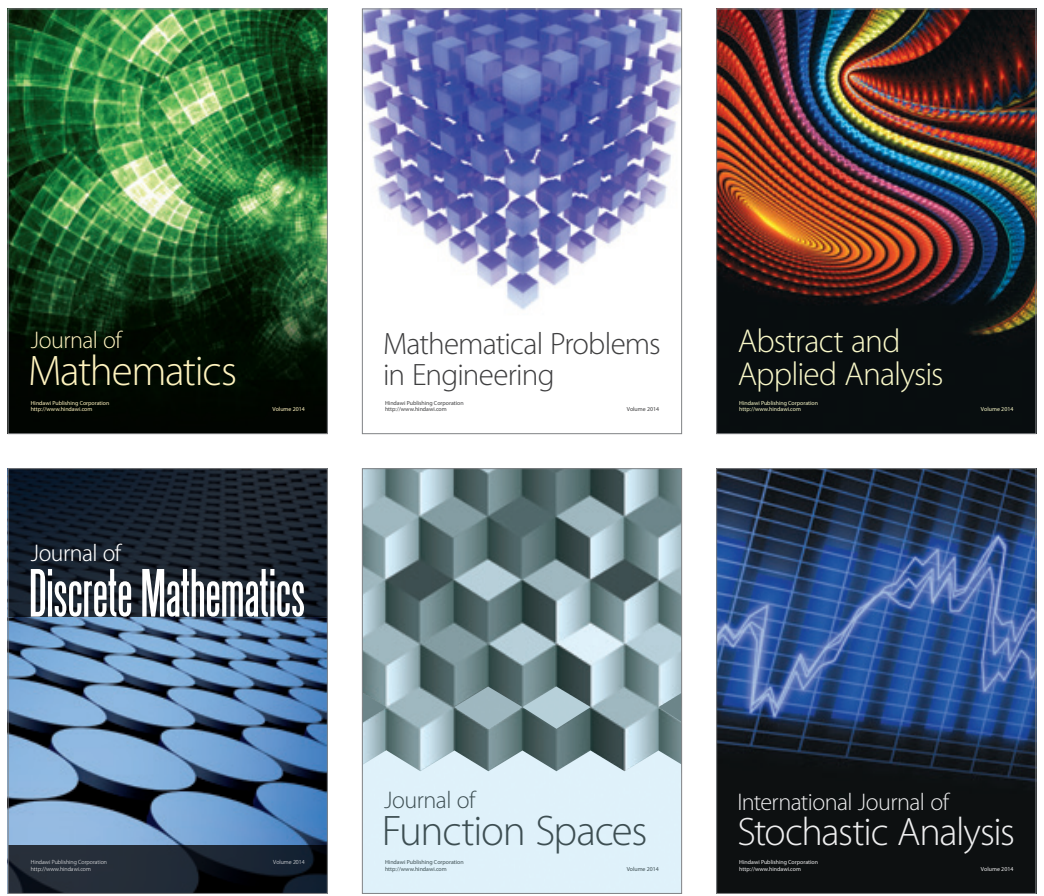

Journal of

Function Spaces

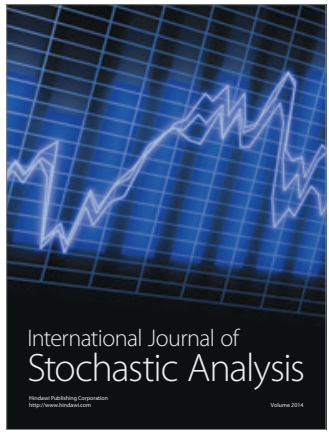

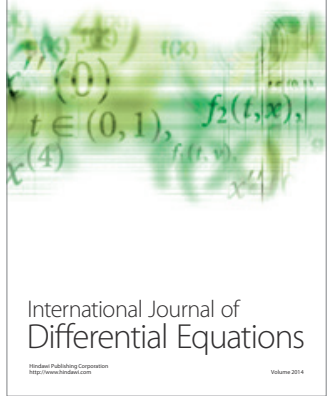
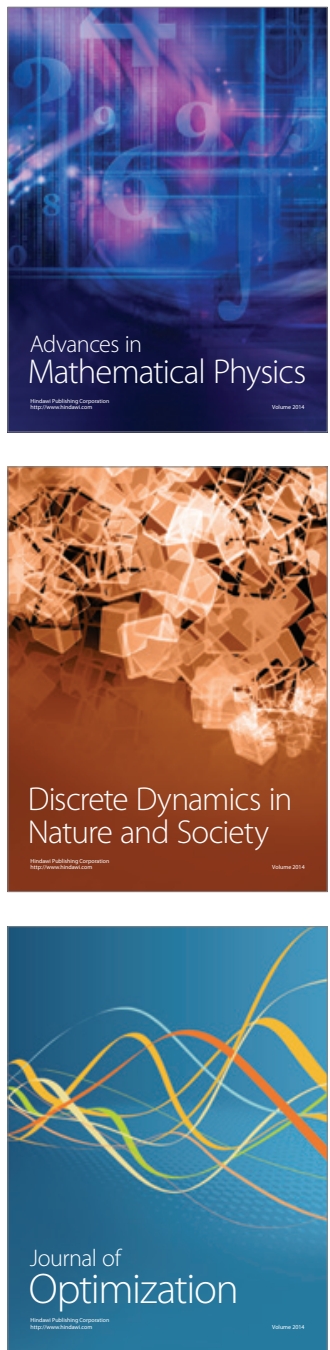